\title{
Effects of Gelidium amansii extracts on in vitro ruminal fermentation characteristics, methanogenesis, and microbial populations
}

\author{
Shin Ja Lee, ${ }^{1, a}$, Nyeon Hak Shin ${ }^{2, a}$, Jin Suk Jeong ${ }^{3}$, Eun Tae Kim, Su Kyoung Lee', \\ II Dong Lee ${ }^{5}$, and Sung Sill Lee ${ }^{1,3, *}$
}

\footnotetext{
* Corresponding Author: Sung Sill Lee Tel: +82-55-772-1883, Fax: +82-55-772-1889

E-mail: Iss@gnu.ac.kr

${ }^{1}$ Institute of Agriculture and Life Science and University-Centered Labs, Gyeongsang National University, Jinju 52828, Korea

2 Livestock Experiment Station, Gyeongsangnamdo Livestock Promotion Research Institute, Sancheong 52733, Korea

${ }^{3}$ Division of Applied Life Science (BK21 program) and Institute of Agriculture \& Life Science (IALS), Gyeongsang National University, Jinju 52828, Korea ${ }^{4}$ National Institute of Animal Science, RDA, Cheonan 31000, Korea

${ }^{5}$ Division of Applied Life Science (BK21), Gyeongsang National University, Jinju 52828, Korea

a These authors contributed equally to the work.
}

ORCID

Shin Ja Lee

https://orcid.org/0000-0002-4224-1211 Nyeon Hak Shin

https://orcid.org/0000-0002-9069-0601

Jin Suk Jeong

https://orcid.org/0000-0001-6877-3067

Eun Tae Kim

https://orcid.org/0000-0001-7486-5638

Su Kyoung Lee

https://orcid.org/0000-0002-0997-2899

Il Dong Lee

https://orcid.org/0000-0003-1221-4383

Sung Sill Lee

https://orcid.org/0000-0002-4621-4333

Submitted Aug 21, 2017; Revised Oct 23, 2017; Accepted Dec 2, 2017
Objective: Gelidium amansii (Lamouroux) is a red alga belonging to the family Gelidaceae and is commonly found in the shallow coasts of many East Asian countries, including Korea, China, and Japan. G. amansii has traditionally been utilized as an edible alga, and has various biological activities. The objective of this study was to determine whether dietary supplementation of G. amansii could be useful for improving ruminal fermentation.

Methods: As assessed by in vitro fermentation parameters such as $\mathrm{pH}$, total gas, volatile fatty acid (VFA) production, gas profile (methane, carbon dioxide, hydrogen, and ammonia), and microbial growth rate was compared to a basal diet with timothy hay. Cannulated Holstein cows were used as rumen fluid donors and $15 \mathrm{~mL}$ rumen fluid: buffer (1:2) was incubated for up to $72 \mathrm{~h}$ with four treatments with three replicates. The treatments were: control (timothy only), basal diet with $1 \%$ G. amansii extract, basal diet with 3\% G. amansii extract, and basal diet with 5\% G. amansii extract.

Results: Overall, the results of our study indicate that G. amansii supplementation is potentially useful for improving ruminant growth performance, via increased total gas and VFA production, but does come with some undesirable effects, such as increasing $\mathrm{pH}$, ammonia concentration, and methane production. In particular, real-time polymerase chain reaction indicated that the methanogenic archaea and Fibrobacter succinogenes populations were significantly reduced, while the Ruminococcus flavefaciens populations were significantly increased at $24 \mathrm{~h}$, when supplemented with G. amansii extracts as compared with controls.

Conclusion: More research is required to elucidate what G. amansii supplementation can do to improve growth performance, and its effect on methane production in ruminants.

Keywords: Gelidium amansii; Growth Rate; In vitro Fermentation; Microbial Growth

\section{INTRODUCTION}

Algae are an economically important and underexploited plant resource, providing potential biomass for animal feed and human foods in recent years. In fact, algae have a broad range of primary metabolites, including polysaccharides, polyphenols, amino acids and minerals [1,2] which can help improve growth rate and feed conversion efficiency [3,4]. Animal nutritionists have been studying manipulation of the rumen microbial ecosystem to reduce methane emission without adverse effects on rumen function. Methane emissions from greenhouse gases are low, but one molecule of methane affects 21 times more than one molecule of carbon dioxide. There is a need to identify feed additives to modify ruminal fermentation characteristics and increase the efficiency of feed utilization, whilst inhibiting ruminal methanogenesis.

Gelidium amansii (G. amansii) is a red alga belonging to the family Gelidaceae and is found in shallow coastal areas of many East Asian countries including North and South Korea, China, and Japan. Moreover, G. amansii has various biological activities, including antioxidant, anti- 
tumor, cytotoxicity and immunomodulation effects $[5,6]$.

Many studies have reported the composition and properties of algae, describing novel functional food ingredients, which may improve animal metabolic function and feed quality [3]. However, few papers have determined the potential of $G$. amansii effects on ruminal fermentation characteristics by in vitro gas production techniques. Such techniques have been applied to study fermentation kinetics relative to feed composition, and allow for rapid screening of a large number of feed additives that may have effects on fermentation process [7]. Previous studies have shown that algae similar to G. amansii are effective in methane reduction $[8,9]$. Therefore, the objective of this study was to determine the potential effects of dietary supplementation of $G$. amansii on in vitro fermentation parameters including total gas production, volatile fatty acid (VFA) composition, gas profile (methane, carbon dioxide, hydrogen, and ammonia) and microbial growth rate, according to changes occurring with microbial diversity, as compared to basal diet with timothy fermentation. The results could help determine whether dietary supplementation of G. amansii could be useful for improving ruminal fermentation.

\section{MATERIALS AND METHODS}

\section{Preparation of G. amansii extract}

Air-dried G. amansii samples were obtained from the Jeju Biodiversity Research Institute (JBRI, Jeju, Korea), and were stored under dry and dark conditions. Washed samples were cut into small pieces, freeze dried and crushed. Crushed powder was extracted with $80 \% \mathrm{EtOH}$ at room temperature, using an ultrasonic cleaner (Branson Ultrasonics corporation, Danbury, CT, USA) and the EtOH eluate solution was filtered through Whatman No. 1 filter paper and concentrated under vacuum.

\section{In vitro fermentation design}

One cannulated Holstein cows $(450 \pm 30 \mathrm{~kg})$ was used as rumen fluid donors and provided with ad libitum access to water and a mineral-vitamin block. Timothy and commercial concentrate ratio $(60: 40, \mathrm{w} / \mathrm{w})$ were fed twice daily at 09:00 and 17:00, equivalent to $2 \%$ of body weight. The rumen fluid collected before morning feeding was strained through four layers of cheese gauze, and the rumen contents were diluted by addition of artificial saliva and maintained at $39^{\circ} \mathrm{C}$. All experimental protocols used in this research were approved and maintained in accordance with the Guide for the Animal Care and Use Committee of Gyeongsang National University (Jinju, Korea).

Dietary treatments were as follows: control (CON), basal diet (without G. amansii extract); treatment 1 (TRT 1), CON+1\% G. amansii extract; treatment 2 (TRT 2), $\mathrm{CON}+3 \%$ G. amansii extract; and treatment 3 (TRT 3), CON+5\% G. amansii extract as basis of substrate (timothy). The chemical composition of timothy was measured to contain the following: amount of moisture, $8.87 \%$; crude protein, $13.37 \%$ ether extracts, $2.25 \%$; crude fiber,
21.87\%; crude ash, $8.62 \%$; neutral detergent fiber, $53.18 \%$; and acid detergent fiber, $30.57 \%$ as percentage of dry matter (DM).

Fifteen $\mathrm{mL}$ of rumen fluid: artificial saliva mixture $(\mathrm{McD}$ ougall, $1: 2, \mathrm{v} / \mathrm{v}$ ) was dispensed into $50 \mathrm{~mL}$ serum bottles, containing $300 \mathrm{mg}$ of timothy for CON and G. amansii extract for TRTs (3 $\mathrm{mg}$ for TRT 1, $9 \mathrm{mg}$ for TRT 2 and $15 \mathrm{mg}$ for TRT 3). The serum bottles were sealed under anaerobic conditions and capped with a butyl rubber stopper with an aluminum cap and then incubated in a gently shaking incubator (Jeio Tech, SI-900R, Daejeon, Korea; $120 \times \mathrm{rpm}$ ) at $39^{\circ} \mathrm{C}$ for $72 \mathrm{~h}$. The in vitro fermentation experiment was a completely randomized block design and performed in triplicate, using 60 serum bottles ( 4 treatments $\times 5$ incubation times $\times 3$ replicates).

\section{Analysis of gas profiles and ruminal fermentation characteristics}

Total gas production was measured according to the method by Theodorous who suggested that the head space gas pressure should be measured using a detachable pressure transducer and a digital readout voltmeter (Laurel Electronics, Inc., Costa Mesa, CA, USA) after removing serum bottles from a shaking incubator. For gas profiling during $72 \mathrm{~h}$, the transducer was connected to the inlet of a disposable Luer-lock three-way stopcock and then gas pressure in the headspace above the culture medium was read from the LED display unit after insertion of a hypodermic syringe needle. Furthermore, another outlet was connected to a gas chromatograph (HP 5890, Agilent Technologies, Santa Clara, CA, USA) using a TCD detector with a Carboxen-1006 Plot capillary column (30 mm×0.53 mm, Supelco, Bellefonte, PA, USA) for detection of methane, carbon dioxide and hydrogen.

Following gas profile analysis, serum bottles were uncapped and the culture medium was subsampled for $\mathrm{pH}$ analysis (MP230, Mettler-Toledo, Columbus, OH, USA), ammonia and VFA concentration. Ammonia concentration was measured as optical density (OD) values using a UV/VIS spectrophotometer (Model 680, Bio-Rad laboratories, Hercules, CA, USA) at $630 \mathrm{~nm}$ after sample preparation. For VFA measurement, culture medium was centrifuged at 3,000 rpm for $3 \mathrm{~min}$ and then supernatants were filtrated using a $0.2 \mu \mathrm{M}$ disposable syringe filter (Whatman Inc., Clifton, NJ, USA). VFA analysis was performed with high performance liquid chromatography (HPLC, Agilent-1200, Waldbronn, Germany) using a UV/VIS detector with a MetaCarb $87 \mathrm{H}$ column (300 mm×7.8 mm, Varian, Palo Alto, CA, USA).

In vitro DM disappearance rate was determined using the nylon bag digestion method. After incubation, the nylon bag containing the substrate was washed twice in a water-bath equipped with a Heidolphs Rotamax 120 (Heidolph Instrument, Nuremberg, Germany) at $100 \mathrm{rpm}$ for $30 \mathrm{~min}$ and then oven dried at $60^{\circ} \mathrm{C}$ to a constant weight. DM disappearance was defined as weight loss before and after incubation in the serum bottle.

Microbial growth performance 
At the end of each fermentation period, samples were centrifuged at 3,000 rpm for $3 \mathrm{~min}$ to remove feed particles and the supernatants were re-centrifuged at 14,000 rpm for $3 \mathrm{~min}$ to pellet. Supernatants were divided for protein and glucose analysis. The protein was evaluated as OD value at $595 \mathrm{~nm}$ measured by a spectrophotometer (Model 680, Bio-Rad Laboratories, USA), using the dye binding properties of Coomassie Blue G-250. For glucose measurement, $200 \mu \mathrm{L}$ of supernatant and $600 \mu \mathrm{L}$ of 3,5-dinitrosalicylic acid solution were mixed and incubated for $5 \mathrm{~min}$ in a boiling water bath. The glucose concentration was determined as OD value at $595 \mathrm{~nm}$ using a microplate reader (Model 680, Bio-Rad Laboratories, USA). After discarding the supernatants, pellets were washed with sodium phosphate buffer ( $\mathrm{pH}$ 6.5) and repeated three more times. The growth rate of total microorganisms was evaluated as OD value at $550 \mathrm{~nm}$ as measured by spectrophotometer (Model 680, Bio-Rad Laboratories, USA).

\section{Quantitative real-time polymerase chain reaction}

Samples were placed in screw-capped tubes containing silica beads for DNA extraction with a high-speed reciprocal shaker, following a modified bead-beating protocol with a Soil kit (Macherey-nagel, Düren, Germany). Briefly, a 1.0-mL aliquot of the incubated culture solution was centrifuged at $3,000 \times \mathrm{rpm}$, and then placed in a NanoDrop Spectrophotometer (Thermo Scientific, Wilmington, DE, USA) to determine nucleic acid concentrations.

Previous reports provided primers and thermocycling protocols used for amplification of general bacteria, ciliate protozoa, methanogenic archaea, Fibrobacter succinogenes (F. succinogenes), Ruminococcus albus ( $R$. albus), and Ruminococcus flavefaciens (R. flavefaciens) were from previously published reports [10-13].

Quantitative real-time polymerase chain reaction (PCR) assays (CFX96 Real-Time system; Bio-rad, USA) were performed with the SYBR Green Supermix (QPK-201, Toyobo Co., LTD., Tokyo, Japan), following Denman and McSweeney [14] and Denman et al [11]. Abundance of these microbes was determined by the following equation:

\section{Relative change in gene expression} $=2^{-\Delta \mathrm{Ct}(\text { Target })-\Delta \mathrm{Ct}(\text { Control })}$
Where Ct represents threshold cycle. All quantitative (q) PCR reaction mixtures (final volume of $20 \mu \mathrm{L}$ ) contained forward and reverse primers, the SYBR Green Supermix and DNA template. Table 1 shown the PCR amplification of the target DNA, included the annealing and the extension temperature, was conducted following the same conditions described in the PCR primer references [10-13].

\section{Statistical analysis}

All experimental data were analyzed using the general linear model procedure of SAS [15] as a completely randomized block design. The effects of supplementation of G. amansii extract on $\mathrm{pH}$, total gas production, DM disappearance, gas profiles, VFA profiles and microbial growth were compared to $\mathrm{CON}$ and significant differences were conducted using polynomial regression to measure the linear and quadratic effects of increasing concentrations of G. amansii extract. Variability in the data was expressed as standard error of the mean and $\mathrm{p}<0.05$ was considered to be statistically significant, whereas $\mathrm{p}<0.10$ was considered a tendency.

\section{RESULTS}

In vitro fermentation characteristics (cumulative $\mathrm{pH}$, gas production, dry matter disappearance rate)

The effect of dose response of G. amansii extract on in vitro fermentation parameters is shown in Table 2. G. amansii extracts increased $\mathrm{pH}$ correlating positively with G. amansii extract concentration. With regards to cumulative gas production by mixed ruminal microorganisms, G. amansii extracts reduced cumulative gas production at $3 \mathrm{~h}$, but increased it at 12,24 and $72 \mathrm{~h}$ as compared with CON, respectively. Lastly, DM disappearance with supplementation of $G$. amansii extracts was lower than that of $\mathrm{CON}$ during the whole experimental period.

Volatile fatty acid profile and acetic acid/propionic acid ratio The effect of dose response of G. amansii extract on VFA profiles of acetate, propionate, butyrate, and acetic acid/propionic acid $(\mathrm{A} / \mathrm{P})$ ratio are shown in Table 3 . The supplementation of G. amansii extract increased total VFA concentration, acetate

Table 1. Polymerase chain reaction primer sets for real-time polymerase chain reaction assays

\begin{tabular}{|c|c|c|}
\hline Target species & Primer sequences $\left(5^{\prime} \rightarrow 3^{\prime}\right)$ & References \\
\hline Ciliate-associated methanogens & $\begin{array}{l}\text { F: GAG CTA ATA CAT GCT AAG GC } \\
\text { R: CCC TCA CTA CAA TCG AGA TTT AAG G }\end{array}$ & Skillman et al [12] \\
\hline Methanogenic archaea & $\begin{array}{l}\text { F: TTC GGT GGA TCD CAR AGR GC } \\
\text { R: GBA RGT CGW AWC CGT AGA ATC C }\end{array}$ & Denman et al [11] \\
\hline Fibrobacter succinogenes & $\begin{array}{l}\text { F: GTT CGG AAT TAC TGG GCG TAA A } \\
\text { R: CGC CTG CCC CTG AAC TAT C }\end{array}$ & Denman and McSweeney [10] \\
\hline Ruminococcus albus & $\begin{array}{l}\text { F: CCC TAA AAG CAG TCT TAG TTC G } \\
\text { R: CCT CCT TGC GGT TAG AAC A }\end{array}$ & Koike and Kobayashi [13] \\
\hline Ruminocuccus flavefaciens & $\begin{array}{l}\text { F: CGA ACG GAG ATA ATT TGA GTT TAC TTA GG } \\
\text { R: CGG TCT CTG TAT GTT ATG AGG TAT TAC C }\end{array}$ & Denman and McSweeney [10] \\
\hline
\end{tabular}


Table 2. Effect of Gelidium amansii extract on in vitro cumulative pH, gas emission, dry matter disappearance by mixed rumen microbial fermentation

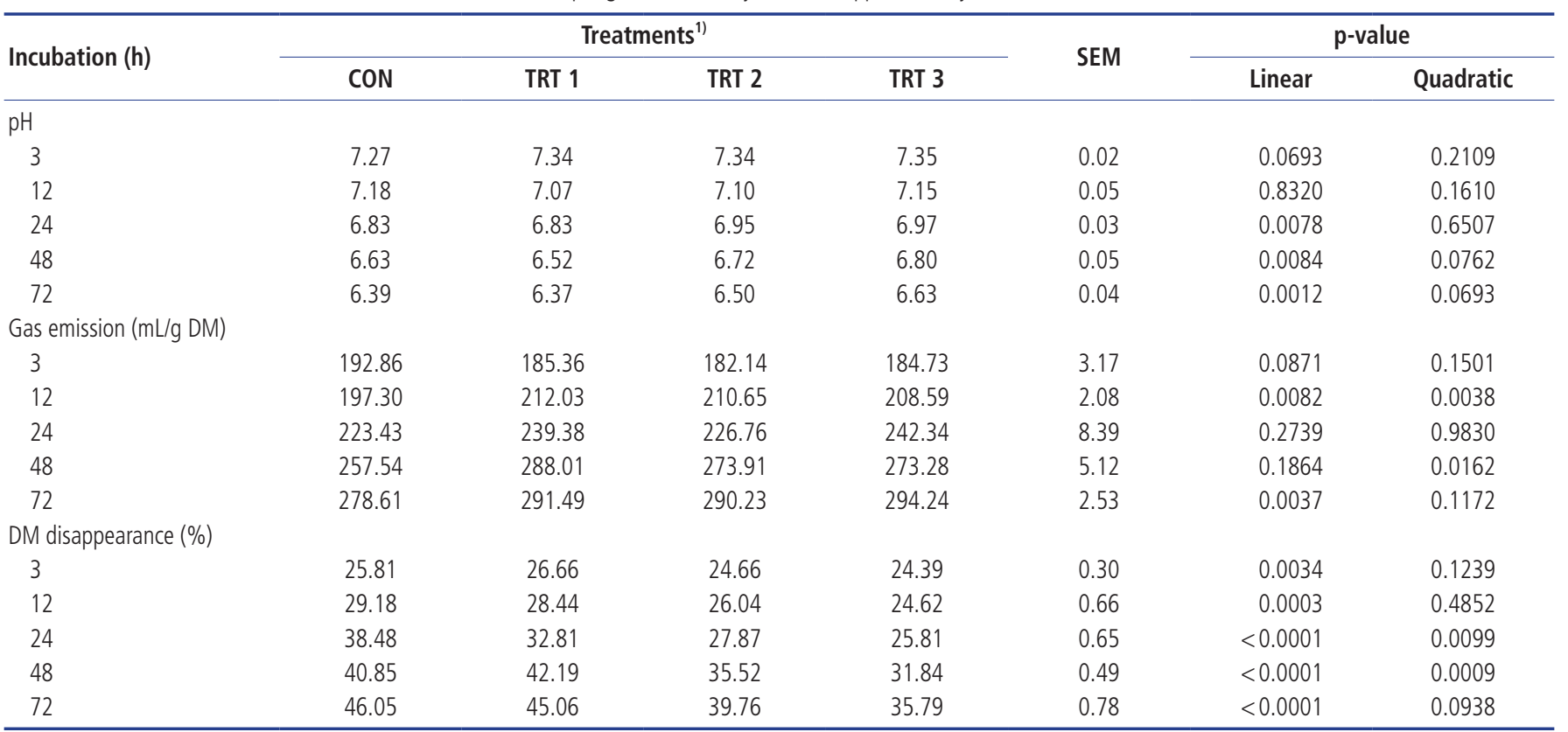

SEM, standard error of the mean; DM, dry matter.

1) Dietary treatments were as follows: CON, basal diet (without G. amansii extract); TRT 1, CON+1\% G. amansii; TRT 2, CON+3\% G. amansii; TRT 3, CON+5\% G. amansii as basis of substrate (timothy).

concentration, propionate concentration, butyrate concentration and $\mathrm{A} / \mathrm{P}$ ratio as compared with $\mathrm{CON}$, respectively.

Emission gas profile (methane, carbon dioxide, and ammonia)

The effect of dose response of G. amansii extract on the gas profiles of methane, carbon dioxide and ammonia are shown in Table 4. G. amansii extract reduced methane emissions relative to $\mathrm{CON}$ at $3 \mathrm{~h}$, carbon dioxide emission was increased relative to $\mathrm{CON}$ at $12 \mathrm{~h}$. Futhermore, G. amansii extract incerased hydrogen, ammonia emission as compared with CON.

\section{In vitro ruminal change in microbial diversity}

The effect of dose response of $G$. amansii extract on microbial growth rate, protein and glucose concentration is shown in Table 5. The supplementation of G. amansii extract increased microbial growth rate and glucose concentration, while reducing protein concentration as compared with $\mathrm{CON}$, respectively.

Changes in ruminal microbial diversity supplemented with G. amansii extracts are shown in Figure 1a (after $12 \mathrm{~h}$ incubation) and Figure $1 \mathrm{~b}$ (after $24 \mathrm{~h}$ incubation), respectively. Supplementation with G. amansii extracts lead to an increase in the ciliateassociated methanogen population at $12 \mathrm{~h}$, while reducing the methanogenic archaea population at $24 \mathrm{~h}$ as compared with $\mathrm{CON}$, respectively. With regards to major fibrolytic microorganisms, G. amansii extracts reduced R. albus at $12 \mathrm{~h}, 24 \mathrm{~h}$ and F. succinogenes at $12 \mathrm{~h}$, while increasing $R$. flavefaciens at $24 \mathrm{~h}$ as compared with CON, respectively.

\section{DISCUSSION}

Overall, G. amansii treatments did not appear to be detrimental to ruminal fermentation as assessed by in vitro fermentation parameters. In fact, G. amansii supplementation has the potential to assist in ruminant feeding for improved gas production and fermentation performance. For example, supplementation with $G$. amansii treatments produced $\mathrm{pH}$ values remaining in the proper range of 6.37 to 7.35 , which is a suitable $\mathrm{pH}$ range for cellulose digestion (6.0 to 6.8), protein synthesis (6.3 to 7.4 ), proteolytic activity (6.5 to 7.0 ) and VFA productivity (6.0 to 6.6 ) as suggested by McCullough [16], as well as for ruminal microbial activity, which is not negatively affected within a $\mathrm{pH}$ range of 5.8 to 7.2 [17]. In addition, \% DM disappearance after G. amansii supplementation was significantly reduced for the whole experimental period; however, total gas production was significantly increased at 12 and $72 \mathrm{~h}$ incubation as compared to CON, which may indicate a potential improvement to feed utilization efficiency [18]. However, when dietary fiber was included in G. amansii treatments, an increase of total gas production was observed without any reduction of \% DM disappearance, which is in agreement with other algae studies involving dietary fiber [19].

In recent years, extensive studies investigating the potential use of terrestrial plants for nutritional manipulation of enteric methane production have been conducted. Interestingly, one study focusing on algae supplementation reported reduced methane production [20]; however, the current study provides evidence that G. amansii supplementation can significantly increase in 
Table 3. Effect of Gelidium amansii extract on total volatile fatty acids (VFA), acetic acid, propionic acid, butyric acid and acetic acid/propionic acid (A/P) ratio by mixed rumen microbial fermentation

\begin{tabular}{|c|c|c|c|c|c|c|c|}
\hline \multirow{2}{*}{ Incubation (h) } & \multicolumn{4}{|c|}{ Treatments $^{1)}$} & \multirow{2}{*}{ SEM } & \multicolumn{2}{|c|}{ p-value } \\
\hline & CON & TRT 1 & TRT 2 & TRT 3 & & Linear & Quadratic \\
\hline \multicolumn{8}{|c|}{ Total VFA concentration (mM/g) } \\
\hline 12 & 83.97 & 97.84 & 98.48 & 100.26 & 2.95 & 0.0056 & 0.0746 \\
\hline 24 & 106.85 & 109.28 & 112.60 & 131.66 & 6.19 & 0.0229 & 0.2161 \\
\hline 48 & 113.99 & 121.27 & 133.06 & 142.44 & 4.36 & 0.0011 & 0.8150 \\
\hline 3 & 49.07 & 51.06 & 59.74 & 64.64 & 2.29 & 0.0006 & 0.5429 \\
\hline 12 & 57.76 & 69.57 & 72.67 & 74.33 & 2.59 & 0.0019 & 0.0859 \\
\hline 24 & 77.48 & 76.22 & 79.42 & 94.10 & 5.78 & 0.0742 & 0.2050 \\
\hline 48 & 81.03 & 84.10 & 93.16 & 100.00 & 4.13 & 0.0072 & 0.6593 \\
\hline 72 & 88.20 & 94.03 & 102.06 & 110.23 & 4.33 & 0.0050 & 0.7937 \\
\hline 48 & 22.70 & 24.45 & 26.58 & 27.65 & 0.66 & 0.0004 & 0.6204 \\
\hline 72 & 24.96 & 29.86 & 31.58 & 34.71 & 0.89 & $<0.0001$ & 0.3488 \\
\hline \multicolumn{8}{|c|}{ Butyric acid concentration (mM/g) } \\
\hline 3 & 8.60 & 8.88 & 9.30 & 9.17 & 0.42 & 0.2873 & 0.6399 \\
\hline 12 & 9.10 & 10.53 & 10.11 & 9.92 & 0.51 & 0.4057 & 0.1543 \\
\hline 24 & 10.03 & 11.75 & 12.01 & 14.53 & 0.50 & 0.0003 & 0.4396 \\
\hline 48 & 10.26 & 12.71 & 13.31 & 14.78 & 0.32 & $<0.0001$ & 0.1595 \\
\hline 72 & 10.64 & 13.06 & 15.13 & 16.92 & 0.56 & $<0.0001$ & 0.5874 \\
\hline \multicolumn{8}{|l|}{ A/P ratio } \\
\hline 3 & 3.49 & 3.49 & 4.11 & 4.38 & 0.22 & 0.0098 & 0.5547 \\
\hline 12 & 3.38 & 3.92 & 4.63 & 4.64 & 0.14 & $<0.0001$ & 0.0895 \\
\hline
\end{tabular}

SEM, standard error of the mean.

1) Dietary treatments were as follows: CON, basal diet (without G. amansii extract); TRT 1, CON+1\% G. amansii; TRT 2, CON+3\% G. amansii; TRT 3, CON+5\% G. amansii as basis of substrate (timothy).

vitro methane and carbon dioxide emission. In particular, increased methane production may have partially been due to an alteration in microbial diversity with an increase in the protozoan population (ciliate-associated methanogens) [21], and a major member of the fibrolytic microorganism population, $R$. flavefaciens [22], resulting from G. amansii treatments as compared with CON. However, methanogenic archaea, R. albus, and F. succinogenes (two other major members of the fibrolytic microorganism population) populations were significantly reduced. Ciliate-associated methanogens may generate up to $37 \%$ of methane produced in the rumen [23]. Therefore, although an increase in ciliate-associated methanogens may help to explain the increase in methane production, a reduction in methanogenic archaea would counter it. With regards to the fibrolytic microorganism population, $R$. flavefaciens normally produces succinic acid as a major fermentation product together with acetic and formic acids, $\mathrm{H}_{2}$, and $\mathrm{CO}_{2}$. Additionally, $R$. albus is a very promising bacterium to produce $\mathrm{H}_{2}$ from energy forage, with the potential of utilizing the cellulosic and hemicellulosic biomass [24]. In contrast, $F$. succinogenes is a non- $\mathrm{H}_{2}$-producing species. The increase in the $R$. flavefaciens population might be the culprit behind the increase in methane and $\mathrm{CO}_{2}$ production. A previous study by Chaucheyras-Durand et al [25] showed that methane production was clearly reduced when the dominant fibrolytic species was a non- $\mathrm{H}_{2}$-producing species, such as $\mathrm{F}$. succinogenes, without significantly impairing fiber degradation and fermentations in the rumen. This was not the case in our study. As such, $\mathrm{H}_{2}$ is of critical concern to the microbial ecosystem in ruminants. $\mathrm{H}_{2}$ produced during enteric fermentation is the precursor of methane emission from ruminants, and the regulation of $\mathrm{H}_{2}$, rather than methane, is the key to controlling ruminant methane emission. In addition, $80 \%$ of total enteric methane production is generated from carbon dioxide and hydrogen as a substrate [26], which supports the positive correlation observed with methane 
Table 4. Effect of Gelidium amansii extract on in vitro methane, carbon dioxide, hydrogen and ammonia emission by mixed rumen microbial fermentation

\begin{tabular}{|c|c|c|c|c|c|c|c|}
\hline \multirow{2}{*}{ Incubation (h) } & \multicolumn{4}{|c|}{ Treatments $^{1)}$} & \multirow{2}{*}{ SEM } & \multicolumn{2}{|c|}{$p$-value } \\
\hline & CON & TRT 1 & TRT 2 & TRT 3 & & Linear & Quadratic \\
\hline \multicolumn{8}{|c|}{ Methane emission (mL/g DM) } \\
\hline 12 & 20.15 & 23.12 & 37.96 & 32.34 & 2.15 & 0.0007 & 0.0811 \\
\hline 24 & 47.37 & 68.32 & 63.34 & 62.90 & 3.40 & 0.0255 & 0.0136 \\
\hline 48 & 72.79 & 87.12 & 74.40 & 70.76 & 5.90 & 0.4959 & 0.1665 \\
\hline 3 & 25.71 & 23.08 & 29.63 & 39.12 & 15.66 & 0.0056 & 0.0121 \\
\hline 12 & 78.06 & 79.89 & 101.93 & 68.73 & 15.00 & 0.0089 & 0.0870 \\
\hline 24 & 119.81 & 151.22 & 114.46 & 113.64 & 22.80 & $<0.0001$ & 0.6923 \\
\hline 48 & 137.75 & 166.10 & 153.13 & 126.46 & 19.07 & $<0.0001$ & 0.4606 \\
\hline 72 & 166.33 & 206.01 & 181.97 & 164.13 & 24.02 & $<0.0001$ & 0.1778 \\
\hline 48 & 12.59 & 14.47 & 12.33 & 12.93 & 0.90 & 0.7888 & 0.4996 \\
\hline 72 & 14.75 & 14.80 & 13.80 & 13.73 & 0.93 & 0.3567 & 0.9506 \\
\hline \multicolumn{8}{|c|}{ Ammonia emission (mg/dL) } \\
\hline 3 & 2.57 & 6.22 & 6.37 & 6.00 & 0.62 & 0.4365 & 0.4623 \\
\hline 12 & 4.52 & 8.76 & 12.23 & 10.80 & 1.45 & 0.1563 & 0.2049 \\
\hline 24 & 4.74 & 10.34 & 21.10 & 25.64 & 1.29 & 0.6709 & 0.0447 \\
\hline 48 & 7.55 & 16.29 & 38.27 & 41.16 & 3.77 & 0.9044 & 0.7609 \\
\hline 72 & 8.17 & 24.00 & 41.41 & 51.31 & 2.00 & 0.0284 & 0.1061 \\
\hline
\end{tabular}

SEM, standard error of the mean; DM, dry matter.

1) Dietary treatments were as follows: CON, basal diet (without G. amansii extract); TRT 1, CON+1\% G. amansii; TRT 2, CON+3\% G. amansii; TRT 3, CON+5\% G. amansii as basis of substrate (timothy).

Table 5. Effect of Gelidium amansii on rumen microbial growth rate, protein and glucose concentration

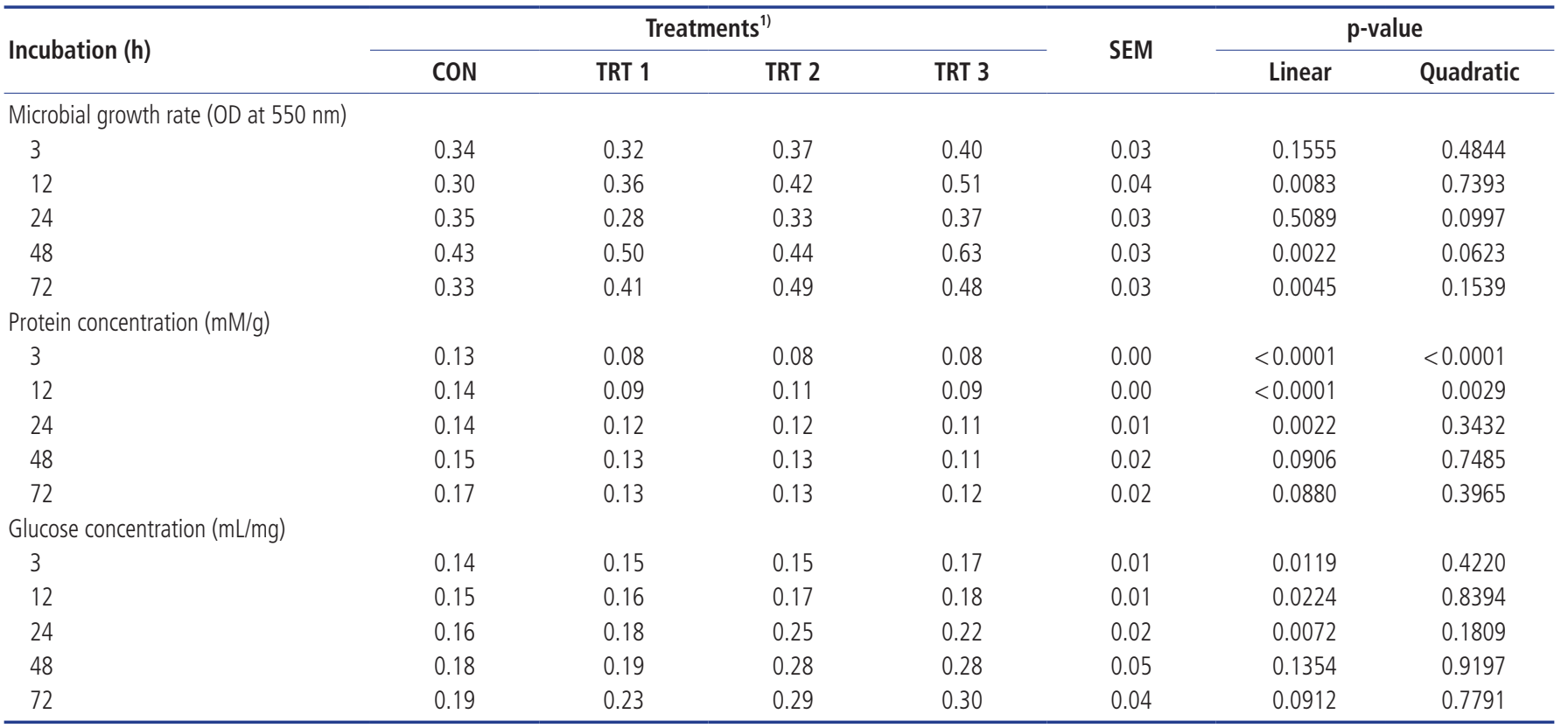

SEM, standard error of the mean; OD, optical density.

1) Dietary treatments were as follows: CON, basal diet (without G. amansii extract); TRT 1, CON+1\% G. amansii; TRT 2, CON+3\% G. amansii; TRT 3, CON+5\% G. amansii as basis of substrate (timothy). 
(a)

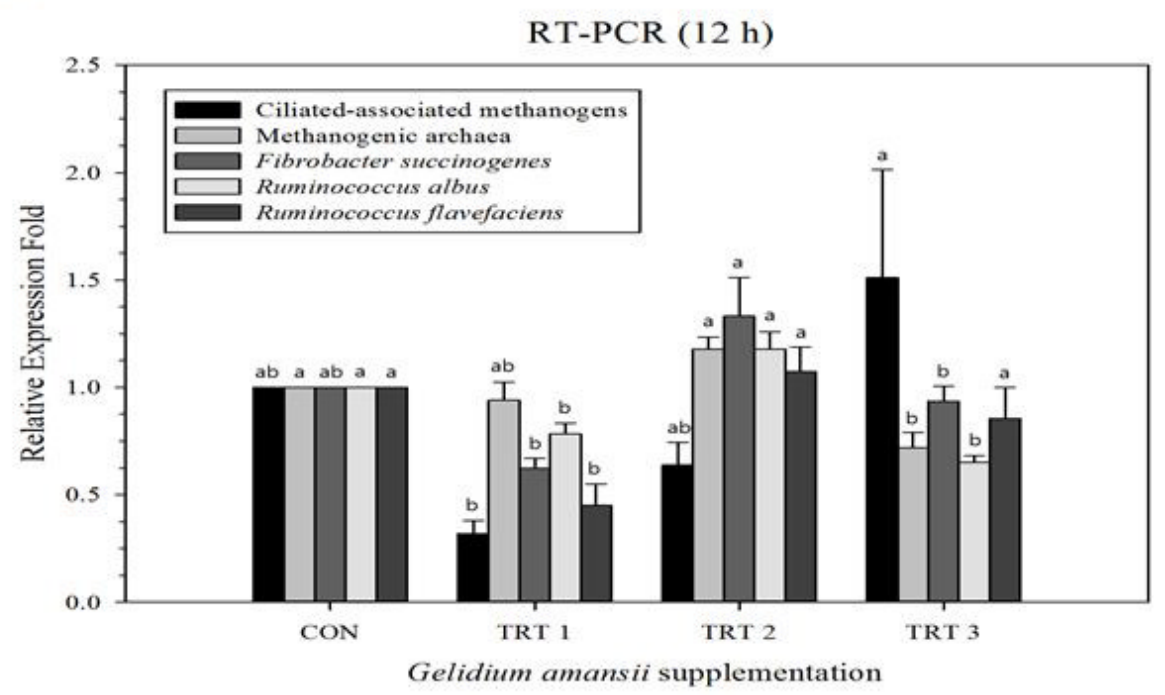

(b)

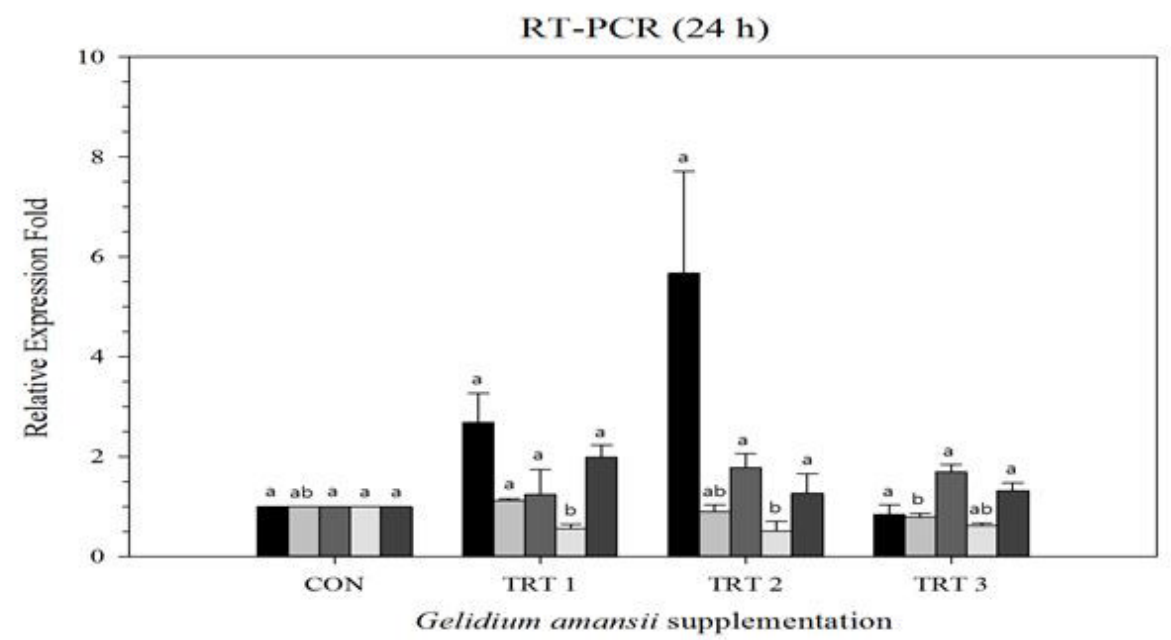

Figure 1. Relative quantification analysis of rumen microorganism populations under in vitro ruminal fermentation by the addition of marine alga Gelidium amansii after $12 \mathrm{~h}$ (a) and $24 \mathrm{~h}$ (b) incubation. Microorganism populations examined include ciliate-associated methanogens, methanogenic archaea, and cellulolytic bacteria (Fibrobacter succinogenes, Ruminococcus albus, and Ruminococcus flavefaciens). Dietary treatments were as follows: CON, basal diet (without G. amansii); TRT 1, CON+1\% G. amansii; TRT 2, CON+3\% G. amansii; TRT 3, CON+5\% G. amansii as basis of substrate (timothy). ${ }^{\text {ab }}$ Means with different superscripts in the same row differ significantly $(p<0.05)$.

and carbon dioxide production in our study. Increased methane emission is indicative of increased methanogen activity. The methanogens utilize mainly hydrogen and carbon dioxide, secondary fermentation products produced by rumen fermentation [27], as well as acetate, as a substrate for methanogenesis [28]. By removing hydrogen as the precursor of ruminal methane emission, methanogens allow the microorganisms involved in fermentation to function optimally and support the complete oxidation of substrates [29]. Overall, G. amansii supplementation resulted in increased methane production, which can be partially explained by increased methanogen activity (increased ciliate-associated methanogens) resulting from an increase in carbon dioxide production by the $R$. flavefaciens populations, acetate concentration and $\mathrm{A} / \mathrm{P}$ ratio; however, all these parameters are still within opti- mal fermentation conditions [30]. In addition to having an effect on methane and carbon dioxide, G. amansii supplementation also resulted in a significantly higher concentration of total VFA, acetate, propionate, butyrate, and $\mathrm{A} / \mathrm{P}$ ratio being produced as compared to $\mathrm{CON}$, demonstrating that fermentation was significantly affected. G. amansii supplementation resulted in a significantly increased amount of microbial growth at 12, 48 and $72 \mathrm{~h}$, as compared to CON, which is in agreement with $\mathrm{Ha}$ et al [18], who suggested that rumen microorganisms need an adaptation period for changing environmental conditions of up to $6 \mathrm{~h}$ before their numbers increase, until nutrient depletion and waste products generated from microbial growth in the medium begin to inhibit their growth. Moreover, VFAs are released as the major end products of rumen microbial fermentation instead of glucose. 
Propionate is the most abundant of the glucogenic acids and the predominant substrate for gluconeogenesis in ruminants [31]. Interestingly, both propionate and glucose concentration were significantly increased throughout the whole experimental period after G. amansii supplementation, demonstrating a positive correlation with one another. Overall, G. amansii supplementation resulted in a higher microbial growth rate, manifesting itself in the form of observed higher total gas and VFA production as compared with CON.

Finally, rumen ammonia concentration can vary depending on the proportion of feed protein and degradation rate. G. amansii supplementation resulted in a significantly higher ammonia concentration during the whole experimental period, which was still within the optimal ammonia concentration range for ruminal fermentation [32], thus demonstrating that rumen fermentation was not detrimentally affected by $G$. amansii supplementation. There was no correlation between ammonia amount and protein production observed in our study.

The objective of this study was to investigate and determine whether dietary supplementation of $G$. amansii could be useful for improving ruminal fermentation, as assessed by in vitro fermentation parameters. Overall, the results of our study indicate that $G$. amansii supplementation is potentially useful (i.e. may improve ruminant growth performance via increased total gas and VFA production), but does come with some undesirable effects. For example, G. amansii supplementation appears to increase methane production (increased methanogenic activity by ciliate-associated methanogens using increased $\mathrm{H}_{2}$ and $\mathrm{CO}_{2}$ being produced by an increased $R$. flavefaciens population), which is in disagreement with previous observations on Rhodophyta supplementation under in vitro fermentation conditions. More research is required to demonstrate and elucidate what G. amansii supplementation can do to improve growth performance and its effect on methane production in ruminants.

\section{CONFLICT OF INTEREST}

We certify that there is no conflict of interest with any financial organization regarding the material discussed in the manuscript.

\section{ACKNOWLEDGMENTS}

This work was carried out with the support of "Cooperative Research Program for Agriculture Science \& Technology Development (Project No. PJ011060)" Rural Development Administration, Republic of Korea. Jin Suk Jeong was supported by Postdoctoral Fellowship from the BK 21+ Program, the Ministry of Education, Science and Technology, Korea. This work was presented as a part of a doctoral dissertation by Nyeon Hak Shin.

\section{REFERENCES}

1.Kim KN, Ahn G, Heo SJ, et al. Inhibition of tumor growth in vitro and in vivo by fucoxanthin against melanoma B16F10 cells. Environ Toxicol Pharmacol 2013:35:39-46.

2.Lee JH, Ko JY, Oh JY, et al. Evaluation of phlorofucofuroeckol-A isolated from Ecklonia cava (Phaeophyta) on anti-lipid peroxidation in vitro and in vivo. Algae 2015;30:313-23.

3.Chowdhury S, Huque K, Khatun M. Algae in animal production. Agracultural science of biodiversity and sustainability workshop. Tune Landboskole, Denmark. 1995. pp. 3-7.

4.MacArtain P, Gill CIR, Brooks M, et al. Nutritional value of edible seaweeds. Nutr Rev 2007;65:535-43.

5.Moller DE, Flier JS. Insulin resistance - mechanisms, syndromes, and implications. N Engl J Med 1991;325:938-48.

6.Yan X, Nagata T, Fan X. Antioxidative activities in some common seaweeds. Plant Foods Hum Nutr 1998;52:253-62.

7.Pell AN, Schofield P. Computerized monitoring of gas production to measure forage digestion in vitro. J Dairy Sci 1993;76:1063-73.

8.Bozic AK, Andrson RC, Carstens GE, et al. Effects of the methaneinhibitors nitrate, nitroethane, lauric acid, Lauricidin and the Hawaiian marine algae Chaetoceros on ruminal fermentation in vitro. Bioresour Technol 2009;100:4017-25.

9.Machado L, Magnusson M, Paul NA, et al. Effects of marine and freshwater macroalgae on in vitro total gas and methane production. PLoS ONE 2014;9:e85289.

10. Denman SE, McSweeney CS. Development of a real-time PCR assay for monitoring anaerobic fungal and cellulolytic bacterial populations within the rumen. FEMS Microbiol Ecol 2006;58:572-82.

11. Denman SE, Tomkins NW, McSweeney CS. Quantitation and diversity analysis of ruminal methanogenic populations in response to the antimethanogenic compound bromochloromethane. FEMS Microbiol Ecol 2007;62:313-22.

12. Skillman LC, Toovey AF, Williams AJ, et al. Development and validation of a real-time PCR method to quantify rumen protozoa and examination of variability between Entodinium populations in sheep offered a hay-based diet. Appl Environ Microbiol 2006;72:200-6.

13. Koike S, Kobayashi Y. Development and use of competitive PCR assays for the rumen cellulolytic bacteria: Fibrobacter succinogenes, Ruminococcus albus and Ruminococcus flavefaciens. FEMS Microbiol Ecol 2001;204:361-6.

14. Denman SE, McSweeney CS. Quantitative (real-time) PCR. In: Makkar HPS, McSweeney CS, editors. Methods in gut microbial ecology for ruminants. Dordrecht, Netherlands: Springer; 2005. pp. 105-15.

15. SAS Institute Inc. SAS/STAT user's guide: Version 9.2 edn. Cary, NC, USA: SAS Institute Inc., 2002.

16. McCullough ME, Sisk LR, Smart WW. Sodium acetate and sodium propionate as additives to all-in-one rations for milk production. J Dairy Sci 1969;52:1605-8.

17. Hiltner P, Dehority B. Effect of soluble carbohydrates on digestion of cellulose by pure cultures of rumen bacteria. Appl Environ Microbiol 1983;46:642-8.

18. Ha JK, Lee SS, Moon YS, et al. Ruminant nutrition and physiology. Seoul, Korea: Seoul National University press; 2005. 
19. Denis C, Morançais M, Li M, et al. Study of the chemical composition of edible red macroalgae Grateloupia turuturu from Brittany (France). J Food Chem 2010;119:913-7.

20. Machad L, Magnusson M, Paul NA, et al. Effects of marine and freshwater macroalgae on in vitro total gas and methane production. PLoS One 2014;9:e85289.

21. Kim ET, Lee SJ, Guan LL, et al. Effects of flavonoid-rich plant extracts on in vitro ruminal methanogenesis, microbial populations and fermentation characteristics. Asian-Australas J Anim Sci 2015;28:530-7.

22. Latham MJ, Wolin MJ. Fermentation of cellulose by Ruminococcus flavefaciens in the presence and absence of Methanobacterium ruminantium. Appl Environ Microbiol 1977;34:297-301.

23. Finlay BJ, Esteban G, Clarke KJ, et al. Some rumen ciliates have endosymbiotic methanogens. FEMS Microbiol Lett 1994;117:157-61.

24. Ntaikou I, Gavala HN, Kornaros M, et al. Hydrogen production from sugars and sweet sorghum biomass using Ruminococcus albus. Int J Hydrogen Energy 2008;33:1153-63.

25. Chaucheyras-Durand F, Masséglia S, Fonty G, et al. Influence of the composition of the cellulolytic flora on the development of hydrogenotrophic microorganisms, hydrogen utilization, and methane production in the rumens of gnotobiotically reared lambs. Appl Environ Microbiol 2010;76:7931-7.
26. Whitman WB, Bowen TL, Boone DR. The methanogenic bacteria. In: The Prokaryotes, 2nd ed A handbook on the biology of bacteria. New Yokr, USA: Springer-Verlag; 1992. pp. 719-67.

27. Jouany JP, Morgavi DP. Use of 'natural' products as alternatives to antibiotic feed additives in ruminant production. Animal 2007;1: 1443-66.

28. Archer DB, Harris JE. Methanogenic bacteria and methane production in various habitats. In: Barnes EM, Mead GC, editors. Anaerobic bacteria in habitats other than man. Oxford, UK: Blackwell Scientific; 1986. pp. 185-223.

29. Martin C, Morgavi DP, Doreau M. Methane mitigation in ruminants: from microbe to the farm scale. Animal 2010;4:351-65.

30. Mitsumori M, Sun W. Control of rumen microbial fermentation for mitigating methane emissions from the rumen. Asian-Australas J Anim Sci 2008;21:144-54.

31. Larsen M, Kristensen NB. Effect of abomasal glucose infusion on splanchnic amino acid metabolism in periparturient dairy cows. J Dairy Sci 2009;92:3306-18.

32. McAllan AB, Sutton JD, Beever DE, et al. Rumen fermentation characteristics and duodenal nutrient flow in lactating dairy cows receiving two types of grass silage with two levels of concentrates. Anim Feed Sci Technol 1994;46:227-35. 